\title{
Increased Homeostasis Model Assessment-Insulin Resistance Is a Risk Factor for Colorectal Adenoma in Japanese Males
}

\author{
Takeshi Sato, ${ }^{1}$ Hiroaki Takeda, ${ }^{2}$ Yu Sasaki ${ }^{1}$ and Sumio Kawata ${ }^{1}$ \\ ${ }^{1}$ Department of Gastroenterology, Yamagata University Faculty of Medicine, Yamagata, Japan \\ ${ }^{2}$ Division of Endoscopy, Yamagata University Hospital, Yamagata, Japan
}

\begin{abstract}
Many previous reports have documented a relationship between metabolic syndrome, in terms of insulin resistance, and colorectal cancer. However, the association of insulin resistance with colorectal adenoma has not been investigated in detail. To elucidate the association of metabolic syndrome components and insulin resistance with adenoma, we investigated homeostasis model assessment insulin resistance (HOMA-IR) in individuals with adenoma. A cross-sectional study was conducted involving individuals who underwent scheduled health examinations using total colonoscopy. Restricting the subjects to males, 261 with adenoma and 702 without adenoma were investigated. HOMA-IR was categorized into three groups: normal $(<1.6)$, intermediate $(\geq 1.6-<2.5)$, and insulin resistance $(2.5 \leq)$. Metabolic syndrome was defined by a combination of any three of the following components: central obesity (waist circumference $\geq 90 \mathrm{~cm}$ ); elevated blood pressure (systolic blood pressure $\geq 130 \mathrm{mmHg}$ and/or diastolic blood pressure $\geq 85 \mathrm{mmHg}$ ); elevated fasting plasma glucose $(\geq 100 \mathrm{mg} / \mathrm{dL})$; reduced high-density lipoprotein-cholesterol $(<40 \mathrm{mg} / \mathrm{dL})$; and elevated triglyceride ( $\geq 150 \mathrm{mg} / \mathrm{dL}$ ). Multivariate analysis of HOMA-IR showed that the intermediate and insulin resistance groups had a significantly increased risk for colorectal adenoma, even after adjustment for waist circumference (odds ratio, 1.62 and 2.23; 95\% confidence interval, 1.07-2.45 and 1.31-3.79, respectively). Accumulation of any metabolic syndrome components increased the risk of colorectal adenoma $(P$ trend $=0.001)$. However, none of the components alone demonstrated a significant risk for colorectal adenoma. Our data indicate that an increased level of HOMA-IR is a risk factor for colorectal adenoma in Japanese males.
\end{abstract}

Keywords: colorectal adenoma; insulin resistance; homeostasis model assessment insulin resistance (HOMA-IR); insulin; metabolic syndrome

Tohoku J. Exp. Med., 2011, 223 (4), 297-303. C 2011 Tohoku University Medical Press

Colorectal cancer is one of the most common cancers worldwide. It has already been shown that obesity (Frezza et al. 2002; Moghaddam et al. 2007), abdominal obesity (Pischon et al. 2006), insulin resistance (Giovannucci 1995; Colangelo et al. 2002, Trevisan et al. 2001) and metabolic syndrome (Cowey and Hardy 2006; Ahmed et al. 2006) are associated with colorectal cancer.

In the late 1990s, colorectal cancer started to increase rapidly in Japan (Yui et al. 2004), and currently it is the third leading cause of cancer death in Japanese males and the leading cause in Japanese females (Center for Control and Information Services, National Cancer Center, Japan). To reduce the incidence and mortality of colorectal cancer, it is necessary to prevent, find, and treat colorectal adenomas, which are the established precursor lesions of colorectal cancer. Therefore, it is important to elucidate the risk factors for colorectal adenoma. Recently, the reported relationship between colorectal cancer and metabolic syndrome has drawn considerable attention (Stocks et al. 2008; Omata et al. 2009). Moreover, some studies have shown that metabolic syndrome is a risk factor for colorectal adenoma (Morita et al. 2005; Kim et al. 2007). Among the components of metabolic syndrome, waist circumference was shown to be that most strongly associated with colorectal adenoma (Kim et al. 2007; Lee et al. 2008), whereas the influences of plasma glucose, lipids and blood pressure appear to vary (Ashbeck et al. 2009; Lui et al. 2010).

Previously, in a study involving a small population, we demonstrated that homeostasis model assessment insulin resistance (HOMA-IR) had a positive association with the incidence of colorectal adenoma (Otake et al. 2005). Unfortunately, only a few subsequent studies have addressed the significance of HOMA-IR as a risk factor for colorectal adenoma (Kang et al. 2010; Yamamoto et al. 2010). Therefore, in the present study, we evaluated the significance of HOMA-IR as a risk factor for colorectal

Received February 3, 2011; revision accepted for publication March 22, 2011. doi: 10.1620/tjem.223.297

Correspondence: Takeshi Sato, M.D., Department of Gastroenterology, Yamagata University Faculty of Medicine, 2-2-2 Iida-Nishi, Yamagata 990-9585, Japan.

e-mail: stakeshi@med.id.yamagata-u.ac.jp 
adenoma in a large population representing that of Japan as a whole. In addition to HOMA-IR, we investigated the relationship of metabolic syndrome and its individual components with colorectal adenoma, to determine which factors are significant.

\section{Methods and Procedures}

\section{Study population}

We studied a consecutive series of subjects who underwent a health examination, including colonoscopy, for school teachers and staff at the Tohoku Central Hospital for Public School Teachers, Yamagata, Japan, between June 2008 and January 2010. This study was approved by the ethics committee of Tohoku Central Hospital. Among the individuals examined during this period, 1,667 agreed to participate in our study. Because 15 participants canceled their colonoscopy, we enrolled 1,652 participants. We then excluded patients for whom any of the following criteria applied: a) a history of colorectal polypectomy $(n=298), \mathrm{b})$ incomplete colonoscopy $(n=$ $31)$, c) history or presence of malignant neoplasms $(n=50)$, d) chronic inflammatory diseases such as collagen disease $(n=6), \mathrm{c})$ inflammatory bowel disease $(n=2)$, e) history of proctocolectomy ( $n$ $=14)$, and f) treatment with insulin $(n=1)$. After the subjects had undergone colonoscopy, the colonoscopists reported the features observed, including the location, size, and number of adenomas, hyperplastic polyps, and other benign lesions. The final diagnosis was checked by another colonoscopist at Tohoku Central Hospital. In this health examination program, endoscopic treatment for colorectal adenoma was not allowed, so we were unable to collect any pathological information about the polyps observed. We assessed only adenomas in this study, because they are well known to be precursors of colorectal cancer. Two were excluded because of incomplete data collection with regard to blood samples and the characteristics of adenomas, respectively. Therefore, a total of 383 participants were excluded. Finally, 963 males and 306 females remained. However, we evaluated only male subjects, who comprised 702 controls and 261 with adenoma, because of the low number of female subjects relative to males, also only 46 women were found to have adenoma.

\section{Covariate assessment}

Participants were encouraged to answer a self-administered questionnaire about alcohol intake, smoking habits, family medical history, past disease, current disease, medication, and history of colorectal polyps or colorectal polypectomy. In this health examination, participants were questioned about smoking status (never, past, current) and daily number of cigarettes consumed, and the interval since cessation of smoking among ever-smokers. Questions about medical history were also asked, including both use of therapeutic and recreational drugs, and previous surgery. Consumption of alcoholic beverages was converted to the amount of ethanol with reference to a previous study (Okamura et al. 2004). The amount of alcohol consumed (g/day) was estimated, and pack-year values calculated for cigarette consumption. One pack-year was defined as the smoking of 20 cigarettes daily for 1 year. We also asked whether participants had a first-degree family history of colorectal cancer.

\section{Anthropometric measurements}

Anthropometric variables were measured by well-trained examiners. Before colonoscopy, body weight and height were measured, and body mass index (BMI) was calculated as the weight in kilo- grams divided by the height in meters squared. Waist circumference was measured in the horizontal plane at the level of the umbilicus. Sitting blood pressure was also measured.

\section{Clinical and laboratory data}

Venous blood samples were drawn after an overnight fast to determine the levels of serum lipids and other biochemical parameters. Serum fasting plasma glucose, hemoglobin A1c (HbA1c), fasting insulin, high-density lipoprotein-cholesterol (HDL-cholesterol), low-density lipoprotein-cholesterol (LDL-cholesterol), triglycerides and uric acid were assayed in the hospital laboratory. HOMA-IR was calculated as the fasting glucose $(\mathrm{mg} / \mathrm{dL}) \times$ fasting insulin $(\mu \mathrm{U} /$ $\mathrm{mL}) / 405$ (Matthews et al. 1985).

In the classification study, HOMA-IR was used to categorize patients into three groups: a normal group $(<1.6)$, an intermediate group $(\geq 1.6-<2.5)$, and a true insulin resistance group $(\geq 2.5)$, according to the criteria proposed by the Japan Diabetes Society (The Japan Diabetes Society 2010).

\section{Diagnostic criteria for metabolic syndrome}

To evaluate the association of metabolic syndrome with adenoma, we used the classification of metabolic syndrome established in 2009 as a result of harmonization of the two common definitions proposed by the American Heart Association/National Heart, Lung and Blood Institute and the International Diabetes Federation (Alberti et al. 2009). This harmonized definition of metabolic syndrome includes a combination of any three of the following components: central obesity (waist circumference higher than $90 \mathrm{~cm}$ ); elevated blood pressure (systolic blood pressure $\geq 130 \mathrm{mmHg}$ and/or diastolic blood pressure $\geq 85 \mathrm{mmHg}$ or treatment for previously diagnosed hypertension); an elevated fasting plasma glucose level $(\geq 100 \mathrm{mg} / \mathrm{dL})$ or receiving drug treatment for elevated glucose; a reduced HDL cholesterol level $(<40 \mathrm{mg} / \mathrm{dL})$ or receiving specific treatment for this disorder; an elevated triglyceride level $(\geq 150 \mathrm{mg} / \mathrm{dL})$ or receiving specific treatment for this disorder. Central obesity was defined on the basis of population- and country-specific criteria, and the cut-off point for central obesity was that for Asians proposed by the International Diabetes Federation Task Force Metabolic Syndrome Criteria 2005 (Alberti et al. 2005).

\section{Statistical analysis}

All statistical analyses were performed using the SPSS version 11.0J software package (SPSS Inc. Tokyo, Japan). Chi-squared test was used for comparison of discrete variables, and the Mann-Whitney $U$-test for comparison of continuous variables. A two-tailed $P$ value of $<0.05$ was considered statistically significant. The continuous variables measured in this study were expressed as the means \pm standard deviation, and categorical variables were expressed as the number (\%). Multivariate analysis was performed using logistic regression and adjusted by age, pack-year value, mean daily alcohol consumption, first-degree familial history of colorectal cancer, and use of therapeutic drugs. For each variable, the odds ratio (OR), 95\% confidence interval $(95 \% \mathrm{CI})$, and $\mathrm{P}$ value were calculated.

\section{Results}

Background characteristics, anthropometric index and biochemical markers

Table 1 shows the characteristics of cases and controls. Individuals with adenoma were significantly older than the 
Table 1. Characteristics of individuals with adenoma and controls.

\begin{tabular}{|c|c|c|c|}
\hline Characteristics & $\begin{array}{l}\text { Controls } \\
(n=702)\end{array}$ & $\begin{array}{l}\text { Individuals with adenoma } \\
\qquad(n=261)\end{array}$ & $P$ value \\
\hline Age (years) & $48.8 \pm 7.1$ & $52.1 \pm 6.0$ & $<0.001$ \\
\hline Familial history of CRC (\%) & $42(6.4)$ & $15(6.3)$ & 1.000 \\
\hline \multicolumn{4}{|l|}{ Cigarette smoking status } \\
\hline Never $(\%)$ & $287(40.9)$ & $147(40.6)$ & 0.125 \\
\hline Former $(\%)$ & $243(34.6)$ & $79(29.1)$ & \\
\hline Current $(\%)$ & $172(24.5)$ & $81(30.3)$ & \\
\hline Smoking (pack-years) & $10.9 \pm 13.0$ & $12.2 \pm 14.4$ & 0.209 \\
\hline Daily alcohol consumption (g/day) & $22.5 \pm 29.6$ & $22.0 \pm 27.5$ & 0.805 \\
\hline Use of anti-hypertensive drugs (\%) & $83(12.1)$ & $39(15.8)$ & 0.153 \\
\hline Use of anti-diabetic drugs (\%) & $22(3.1)$ & $11(4.2)$ & 0.426 \\
\hline Use of lipid disorder treatment (\%) & $56(8.0)$ & $28(11.1)$ & 0.155 \\
\hline Use of Aspirin/NSAIDs (\%) & $18(2.6)$ & $6(2.3)$ & 1.000 \\
\hline $\operatorname{BMI}\left(\mathrm{kg} / \mathrm{m}^{2}\right)$ & $24.5 \pm 3.0$ & $25.1 \pm 3.5$ & 0.017 \\
\hline $\mathrm{WC}(\mathrm{cm})$ & $86.8 \pm 7.9$ & $88.6 \pm 9.5$ & 0.012 \\
\hline SBP (mmHg) & $121.6 \pm 15.3$ & $124.0 \pm 15.1$ & 0.020 \\
\hline $\mathrm{DBP}(\mathrm{mmHg})$ & $77.2 \pm 9.7$ & $78.5 \pm 9.8$ & 0.059 \\
\hline FPG (mg/dl) & $93.1 \pm 31.8$ & $97.8 \pm 26.8$ & $<0.001$ \\
\hline HbA1c (\%) & $5.3 \pm 0.6$ & $5.5 \pm 0.9$ & $<0.001$ \\
\hline Insulin $(\mu \mathrm{U} / \mathrm{ml})$ & $5.9 \pm 4.3$ & $7.2 \pm 6.1$ & $<0.001$ \\
\hline HOMA-IR & $1.4 \pm 1.1$ & $1.8 \pm 2.1$ & $<0.001$ \\
\hline HDL-c (mg/dl) & $59.0 \pm 14.9$ & $57.4 \pm 14.1$ & 0.167 \\
\hline LDL-c (mg/dl) & $130.2 \pm 31.8$ & $130.3 \pm 32.3$ & 0.835 \\
\hline Triglycerides (mg/dl) & $162.9 \pm 106.1$ & $173.5 \pm 96.8$ & 0.020 \\
\hline Uric acid (mg/dl) & $6.2 \pm 1.2$ & $6.2 \pm 1.3$ & 0.912 \\
\hline
\end{tabular}

CRC, colorectal cancer; BMI, body mass index; WC, waist circumference; SBP, systolic blood pressure; DBP, diastolic blood pressure; FPG, fasting plasma glucose; HDL-c, high-density lipoprotein-cholesterol; LDL-c, low-density lipoprotein-cholesterol. The $P$ value was given by the chi-squared test or the MannWhitney $U$-test.

controls. However, familial history of colorectal cancer, pack-year value, and mean daily alcohol consumption did not differ significantly between the groups. The rate of drug use also showed no significant inter-group difference. Individuals with adenoma had a significantly higher body mass index, waist circumference, systolic blood pressure, fasting plasma glucose level, HbAlc, insulin, HOMA-IR, and triglycerides.

\section{Odds ratios for the three categories of HOMA-IR}

Table 2 shows division of the patients into three categories based on HOMA-IR to evaluate the risk of colorectal adenoma. The 1.5-2.4 (intermediate) and $\geq 2.5$ (true resistance) categories of HOMA-IR were associated with a significant risk even after adjustment for age, familial history of colorectal adenoma, pack-year value, mean daily ethanol consumption, treatment for diabetes mellitus, use of aspirin/ non-steroidal anti-inflammatory drugs (NSAIDs), and waist circumference (OR, 1.61; 95\% CI, 1.06-2.44; $P$ value, 0.024 and OR, 2.22; 95\% CI, 1.30-3.77; $P$ value, 0.003 , respectively).
HOMA-IR, glucose, and insulin characteristics of individuals with adenoma

We evaluated HOMA-IR, glucose, and insulin in relation to characteristics of adenoma. HOMA-IR was slightly higher in individuals with adenoma in both the right and left sides of the colon than in either side of the colon alone, and with an adenoma size exceeding $10 \mathrm{~mm}$, but the differences were not significant. The number of adenomas was not related to HOMA-IR. The fasting plasma glucose level was not associated with adenoma location, size or number. However, the fasting insulin level was significantly higher in individuals with large adenomas.

\section{Metabolic syndrome-associated colorectal adenoma}

Table 3 shows the various components of the harmonized metabolic syndrome criteria for colorectal adenoma. When the individual components were analyzed separately, none demonstrated a significant association with colorectal adenoma risk. The subjects with metabolic syndrome had a significantly higher risk for colorectal adenoma, as shown in Table 4 (OR, 1.67; 95\% CI, 1.19-2.36; $P$ value, 0.003). 
Table 2. Association of the three categories of HOMA-IR with colorectal adenoma.

\begin{tabular}{|c|c|c|c|c|c|c|c|c|}
\hline \multirow{2}{*}{ HOMA-IR } & \multirow{2}{*}{$\begin{array}{c}\text { Control } \\
(n=702)\end{array}$} & \multirow{2}{*}{$\begin{array}{c}\text { Adenoma } \\
(n=261)\end{array}$} & \multicolumn{3}{|c|}{ Univariate } & \multicolumn{3}{|c|}{ Multivariate $^{\text {}}$} \\
\hline & & & OR & 95\% C.I. & $P$ value & OR & 95\% C.I. & $P$ value \\
\hline Normal $(<1.6)$ & $502(71.5)$ & $151(57.8)$ & 1.00 & ref & & 1.00 & ref & \\
\hline Intermediate $(\geq 1.6-<2.4)$ & $128(18.2)$ & $66(25.3)$ & 1.71 & $(1.21-2.43)$ & 0.002 & 1.62 & $(1.07-2.45)$ & 0.003 \\
\hline Insulin resistance $(\geq 2.5)$ & $72(10.3)$ & $44(16.9)$ & 2.03 & $(1.34-3.08)$ & 0.001 & 2.23 & $(1.31-3.79)$ & $<0.001$ \\
\hline$P_{\text {trend }}$ & & & $<0.001$ & & & 0.001 & & \\
\hline
\end{tabular}

Ж adjusted for age, pack-years, daily alcohol consumption, familial history of colorectal cancer, use of diabetes mellitus treatment, use of aspirin/NSAIDs, and waist circumference. The $P$ trend was evaluated by entering it into the model as a single variable.

Table 3. Association of the components of the harmonized metabolic syndrome criteria (2009) with the risk for colorectal adenoma.

\begin{tabular}{|c|c|c|c|c|c|c|c|c|}
\hline \multirow{2}{*}{ Variables } & \multirow{2}{*}{$\begin{array}{c}\text { Control } \\
(n=702)\end{array}$} & \multirow{2}{*}{$\begin{array}{c}\text { Adenoma } \\
(n=261)\end{array}$} & \multicolumn{3}{|c|}{ Univariate } & \multicolumn{3}{|c|}{ Multivariate $^{\dagger}$} \\
\hline & & & OR & 95\% C.I. & $P$ value & OR & 95\% C.I. & $P$ value \\
\hline Central obesity & $272(38.8)$ & $101(38.7)$ & 1.00 & $(0.74-1.33)$ & 0.976 & 0.97 & $(0.70-1.35)$ & 0.861 \\
\hline Elevated BP & $256(36.5)$ & $80(30.7)$ & 0.77 & $(0.57-1.04)$ & 0.090 & 0.72 & $(0.51-1.02)$ & 0.065 \\
\hline Elevated FPG & $125(17.8)$ & $43(16.5)$ & 0.91 & $(0.62-1.33)$ & 0.622 & 0.95 & $(0.62-1.45)$ & 0.815 \\
\hline Reduced HDL-c & $282(40.2)$ & $107(41.0)$ & 1.03 & $(0.77-1.38)$ & 0.829 & 1.12 & $(0.80-1.58)$ & 0.518 \\
\hline Elevated TG & $91(13.0)$ & 31 (11.9) & 0.90 & $(0.59-1.40)$ & 0.647 & 0.95 & $(0.57-1.57)$ & 0.836 \\
\hline
\end{tabular}

BP, blood pressure; FPG, fasting plasma glucose; HDL-c, high-density lipoprotein-cholesterol; TG, triglyceride. $\dagger$ analyzed with all components and adjusted for age, smoking status, daily alcohol consumption, and familial history of colorectal cancer.

Table 4. Association of the number of harmonized metabolic syndrome criteria (2009) with the risk of colorectal adenoma.

\begin{tabular}{|c|c|c|c|c|c|c|c|c|}
\hline \multirow{2}{*}{ Variables } & \multirow{2}{*}{$\begin{array}{c}\text { Control } \\
(n=702)\end{array}$} & \multirow{2}{*}{$\begin{array}{l}\text { Adenoma } \\
(n=261)\end{array}$} & \multicolumn{3}{|c|}{ Univariate } & \multicolumn{3}{|c|}{ Multivariate } \\
\hline & & & OR & 95\% C.I. & $P$ value & OR & 95\% C.I. & $P$ value \\
\hline MS diagnosis & $145(20.7)$ & $86(33.0)$ & 1.89 & $(1.38-2.59)$ & $<0.001$ & 1.67 & $(1.19-2.36)$ & 0.003 \\
\hline \multicolumn{9}{|l|}{ MS risk number } \\
\hline 0 & $211(30.1)$ & $56(21.5)$ & 1.00 & ref & & 1.00 & ref & \\
\hline 1 & $201(28.6)$ & $59(22.6)$ & 1.02 & $(0.73-1.67)$ & 0.633 & 1.04 & $(0.67-1.61)$ & 0.876 \\
\hline 2 & 145 (20.7) & $60(23.0)$ & 1.56 & $(1.02-2.38)$ & 0.039 & 1.45 & $(0.91-2.29)$ & 0.115 \\
\hline 3 & $93(13.2)$ & $50(19.1)$ & 2.04 & $(1.29-3.19)$ & 0.002 & 1.69 & $(1.03-2.77)$ & 0.036 \\
\hline$\geq 4$ & $52(7.4)$ & $36(13.8)$ & 2.61 & $(1.56-4.38)$ & $<0.001$ & 2.23 & $(1.28-3.88)$ & 0.005 \\
\hline$P_{\text {trend }}$ & & & $<0.001$ & & & 0.001 & & \\
\hline
\end{tabular}

\$ adjusted for age, smoking status, alcohol intake, and familial history of colorectal cancer. The $P$ trend was evaluated by entering it into the model as a single variable.

Subjects who had more than three or four components of metabolic syndrome had a higher risk, and the risk was increased in patients with three or four compared with those who had none (OR, 1.69; 95\% CI, 1.03-2.77; $P$ value, 0.036 and OR 2.23; 95\% CI 1.28-3.88; $P$ value, 0.005 , respectively, $P$ trend $=0.001$ ).

\section{Discussion}

The HOMA-IR was proposed on the basis of the results of the normal glucose clump test, the product of the fasting plasma glucose level and the insulin level being inversely associated with glucose intake (Matthews et al. 1985). This index is generally used to reflect insulin resistance, and is thought to be the most useful index for health assessment in a large number of subjects. Previously, many studies have evaluated the relationship between colorectal adenoma and glucose metabolism-related factors such as hyperglycemia, hyperinsulinemia, increased levels of insulin-like growth factor (IGF)-1, and decreased levels of IGF binding protein-3 (Giovannucci et al. 2000; Renehan et al. 2001; Marugame et al. 2002). In the present study, we revealed that HOMA-IR was a significant risk factor for 
colorectal adenoma, and moreover this represented the first application of HOMA-IR categorized according to the Japanese insulin resistance classification. The OR of the true resistance group relative to the normal group was 2.23. Kang et al. (2010) reported that the OR of the highest HOMA-IR group relative to the lowest group in five categories was increased significantly to 1.99 . In contrast, Yamamoto et al. (2010) reported that the mean value of HOMR-IR in patients with adenoma was not different from that in controls, and that HOMA-IR was not a risk factor for colorectal adenoma.

We also evaluated the relationship of HOMA-IR to the location, size and number of colorectal adenomas. Individuals with adenomas over $10 \mathrm{~mm}$ in size showed a significantly higher insulin level, but not a higher HOMA-IR. Adenomas learned toward $<10 \mathrm{~mm},>10 \mathrm{~mm}$ group was so small. This might have accounted for the large difference in the insulin level between the two groups. However, it is known that an increased insulin level prevents apoptosis of colorectal tumors (Keku et al. 2005). As the present study did not include a pathological assessment of colorectal adenomas, we were unable to investigate the relationship between HOMA-IR and advanced adenomas, which has been described as colorectal polyps $>10 \mathrm{~mm}$ in diameter and/or villous components and/or severe dysplasia, which have high malignant potential (Winawer et al. 1993; Zarchy and Ershoff 1994). Therefore our evaluation was limited to only the size of advanced adenomas.

The present study also revealed that metabolic syndrome is a risk factor for colorectal adenoma. The OR for patients with metabolic syndrome relative to controls was 1.67 , which was similar to the values reported in other studies (Morita et al. 2005; Kim et al. 2007; Lui et al. 2010). In addition to metabolic syndrome itself, Wang et al. (2005) reported that accumulation of a number of components of metabolic syndrome led to an increasing risk of colorectal adenoma, according to the NCEP-ATPIII diagnostic criteria. Their evaluation was conducted using rectosigmoidscopy. However, Lee et al. (2008) obtained similar results using total colonoscopy in male subjects. In contrast, Tsilidis et al. (2010) did not find any relationship between an accumulated number of metabolic syndrome components and an increased risk of adenoma. However, their evaluation was a cohort study, and the population was relatively small, including 132 controls and 260 individuals with adenoma. In the present study we clearly revealed that an accumulated number of components of metabolic syndrome were associated with an increased risk of colorectal adenoma.

We were also interested in which component(s) of metabolic syndrome most strongly influenced the risk of colorectal adenoma. Most studies have reported that waist circumference is a significant risk factor in itself (Martikainen and Marmot 1999; Kim et al. 2007; Ashbeck et al. 2009; Nam et al. 2010; Yamamoto et al. 2010). However, we did not find any significant relationship with waist circumference in males. Although we applied the Asian criteria for waist circumference ( $\geq 90 \mathrm{~cm}$ ) (Alberti et al. 2005), we also applied the Japanese criteria ( $\geq 85 \mathrm{~cm}$ ) (Japanese Committee of the Metabolic Syndrome Diagnostic Criteria 2005) and obtained similar results (data not shown). Kang and Sass investigated visceral fat area as a significant risk factor for colorectal adenoma, and also reported that waist circumference and subcutaneous fat did not show a constant relationship (Kang et al. 2010; Sass et al. 2004). Tsilidis also reported that there was no relationship between BMI and adenoma in their cohort study (Tsilidis et al. 2010). Although the relationship between visceral fat area and colorectal adenoma appears to be undeniable, any association with waist circumference, subcutaneous fat or BMI remains to be clarified.

Almost all of the subjects evaluated in this study were teachers working in public schools. Some previous studies have demonstrated an inverse association of obesity with education status (Kahn and Williamson 1990; Lee et al. 2005). Therefore, the high education status of the present population might have affected their waist circumference. Other than waist circumference, none of the other components of metabolic syndrome was extracted as a significant risk factor by logistic regression analysis. These results indicate that each of the components of metabolic syndrome had a weak association with colorectal adenoma. However, the risk for colorectal adenoma increased as more of these components accumulated.

In conclusion, although our study was restricted to males, we confirmed that metabolic syndrome is a significant risk factor for colorectal adenoma. Moreover, we revealed that an increased HOMA-IR value was also a significant risk factor for colorectal adenoma, surpassing any of the components of metabolic syndrome. The OR for HOMA-IR was comparable to that for metabolic syndrome. Hereafter, HOMA-IR should be considered for large-scale screening for colorectal adenoma risk, as well as metabolic syndrome.

\section{Conflict of Interest}

All authors have no conflict of interest.

\section{References}

Ahmed, R.L., Schmitz, K.H., Anderson, K.E., Rosamond, W.D. \& Folsom, A.R. (2006) The metabolic syndrome and risk of incident colorectal cancer. Cancer, 107, 28-36.

Alberti, K.G., Eckel, R.H., Grundy, S.M., Zimmet, P.Z., Cleeman, J.I., Donato, K.A., Fruchart, J.C., James, W.P., Loria, C.M. \& Smith, S.C. Jr. (2009) Harmonizing the Metabolic Syndrome: A Joint Interim Statement of the International Diabetes Federation Task Force on Epidemiology and Prevention; National Heart, Lung, and Blood Institute; American Heart Association: World Heart Federation; International Atherosclerosis Society; and International Association for the Study of Obesity. Circulation, 120, 1640-1645.

Alberti, K.G., Zimmet, P. \& Shaw, J.; IDF Epidemiology Task Force Consensus Group. (2005) The metabolic syndrome: a new worldwide definition. Lancet, 366, 1059-1062. 
Ashbeck, E.L., Jacobs, E.T., Martinez, M.E., Gerner, E.W., Lance, P. \& Thompson, P.A. (2009) Components of metabolic syndrome and metachronous colorectal neoplasia. Cancer Epidem. Biomar., 18, 1134-1143.

Colangelo, L.A., Gapstur, S.M., Gann, P.H., Dyer, A.R. \& Liu, K. (2002) Colorectal cancer mortality and factors related to the insulin resistance syndrome. Cancer Epidem. Biomar., 11, 385-391.

Cowey, S. \& Hardy, R.W. (2006) The metabolic syndrome: a highrisk state for cancer? Am. J. Pathol., 169, 1505-1522.

Frezza, E.E., Wachtel, M.S. \& Chiriva-Internati. M. (2002) Influence of obesity on the risk of developing colon cancer. Gut, 55, 285-291.

Giovannucci, E. (1995) Insulin and colon cancer. Cancer Cause Control, 6, 164-179.

Giovannucci, E., Pollak, M.N., Platz, E.A., Willett, W.C., Stampfer, M.J., Majeed, N., Colditz, G.A., Speizer, F.E. \& Hankinson, S.E. (2000) A prospective study of plasma insulin-like growth factor-1 and binding protein-3 and risk of colorectal neoplasia in women. Cancer Epidem. Biomar., 9, 345-349.

Japanese Committee of the Metabolic Syndrome Diagnostic Criteria (2005) Definition and diagnostic criteria of metabolic syndrome. J. Jpn. Soc. Int. Med., 94, 188-203. (in Japanese)

Kahn, H.S. \& Williamson, D.F. (1990) The contributions of income, education and changing marital status to weight change among US men. Int. J. Obesity, 14, 1057-1068.

Kang, H.W., Kim, D., Kim, H.J., Kim, C.H., Kim, Y.S., Park, M.J., Cho, S.H., Sung, M.W., Jung, H.C., Lee, H.S. \& Song, I.S. (2010) Visceral obesity and insulin resistance as risk factors for colorectal adenoma: a cross-sectional, case-control study. Am. J. Gastroenterol., 105, 178-187.

Keku, T.O., Lund, P.K., Galanko, J., Simmons, J.G, Woosley, J.T. \& Sandler, R.S. (2005) Insulin resistance, apoptosis and colorectal adenoma risk. Cancer Epidem. Biomar., 14, 20762081.

Kim, J.H., Lim, Y.J., Kim, Y.H., Sung, I.K., Shim, S.G., Oh, S.O., Park, S.S., Yang, S., Son, H.J., Rhee, P.L., Kim, J.J., Rhee, J.C. \& Choi, Y.H. (2007) Is metabolic syndrome a risk factor for colorectal adenoma? Cancer Epidem. Biomar., 16, 15431546.

Lee, G.E., Park, H.S., Yun, K.E., Jun, S.H., Kim, H.K., Cho, S.I. \& Kim, J.H. (2008) Association between BMI and metabolic syndrome and adenomatous colonic polyps in Korean men. Obesity, 16, 1434-1439.

Lee, W.Y., Jung, C.H., Park, J.S., Rhee, E.J. \& Kim, S.W. (2005) Effects of smoking, alcohol, exercise, education, and family history on the metabolic syndrome as defined by the ATP III. Diabetes Res. Clin. Pr., 67, 70-77.

Lui, C.S., Hsu, H.S., Li, C.I., Jan, C.I., Li, T.C., Lin, W.Y., Lin, T., Chen, Y.C. \& Lee, C.C. (2010) Central obesity and atherogenic dyslipidemia in metabolic syndrome are associated with increased risk for colorectal adenoma in a Chinese population. B.M.C. Gastroenterol., 10(51).

Martikainen, P.T. \& Marmot, M.G. (1999) Socioeconomic differences in weight gain and determinants and consequences of coronary risk factors. Am. J. Clin. Nutr., 69, 719-726.

Marugame, T., Lee, K., Eguchi, H., Oda, T., Shinchi, K. \& Kono, S. (2002) Relation of impaired glucose tolerance and diabetes mellitus to colorectal adenomas in Japan. Cancer Cause Control, 13, 917-921.

Matthews, D.R., Hosker, J.P., Rudenski, A.S., Naylor, B.A., Treacher, D.F. \& Turner, R.C. (1985) Homeostasis model assessment: insulin resistance and beta-cell function from fasting plasma glucose and insulin concentrations in man. Diabetologia, 28, 412-419.

Moghaddam, A.A., Woodward, M. \& Huxley, R. (2007) Obesity and risk of colorectal cancer: a meta-analysis of 31 studies with 70000 events. Cancer Epidem. Biomar., 16, 2533-2547.

Morita, T., Tabata, S., Mineshita, M., Mizoue, T., Moore, M.A. \&
Kono, S. (2005) The metabolic syndrome is associated with increased risk of colorectal adenoma development: the SelfDefense Forces health study. Asian Pac. J. Cancer P., 6, 485-489.

Nam, S.Y., Kim, B.C., Han, K.S., Ryu, K.H., Park, B.J., Kim, H.B. \& Nam, B.H. (2010) Abdominal visceral adiopse tissue predicts risk of colorectal adenoma in both sexes. Clin. Gastroenterol. H., 8, 443-450.

Okamura, T., Tanaka, T., Yoshita, K., Chiba, N., Takebayashi, T., Kikuchi, Y., Tamaki, J., Tamura, U., Minai, J., Kadowaki, T., Miura, K., Nakagawa, H., Tanihara, S., Okayama, A. \& Ueshima, H.: HIPOP-OHP research group. (2004) Specific alcoholic beverage and blood pressure in a middle-aged Japanese population: the High-risk and Population Strategy for Occupational Health Promotion (HIPOP-OHP) Study. J. Hum. Hypertens., 18, 9-16.

Omata, F., Brown, W.R., Tokuda, Y., Takahashi, O., Fukui, T., Ueno, F. \& Mine, T. (2009) Modifiable risk factors for colorectal neoplasms and hyperplastic polyps. Intern. Med., 48, 123-128

Otake, S., Takeda, H., Suzuki, Y., Fukui, T., Watanabe, S., Ishihama, K., Saito, T., Togashi, H., Nakaumura, T., Matsuzawa, Y. \& Kawata, S. (2005) Association of visceral fat accumulation and plasma adiponectin with colorectal adenoma: evidence for participation of insulin resistance. Clin. Cancer Res., 15, 3642-3646.

Pischon, T., Lahmann, P.H., Boeing, H., Friedenreich, C., Norat, T., Tjønneland, A., Halkjaer, J., Overvad, K., Clavel-Chapelon, F., Boutron-Ruault, M.C., Guernec, G., Bergmann, M.M., Linseisen, J., Becker, N., Trichopoulou, A., Trichopoulos, D., Sieri, S., Palli, D., Tumino, R., Vineis, P., Panico, S., Peeters, P.H., Bueno-de-Mesquita, H.B., Boshuizen, H.C., Van Guelpen, B., Palmqvist, R., Berglund, G., Gonzalez, C.A., Dorronsoro, M., Barricarte, A., Navarro, C., Martinez, C., Quirós, J.R., Roddam, A., Allen, N., Bingham, S., Khaw, K.T., Ferrari, P., Kaaks, R., Slimani, N. \& Riboli, E. (2006) Body size and risk of colon and rectal cancer in the European Prospective Investigation Into Cancer and Nutrition (EPIC). $J$. Natl. Cancer Inst., 98, 920-931.

Renehan, A.G., Painter, J.E., Atkin, W.S., Potten, C.S., Shalet, S.M. \& O'Dwyer, S.T. (2001) High-risk colorectal adenomas and serum insulin-like growth factors. Br. J. Surg., 88, 107-113.

Sass, D.A., Schoen, R.E., Weissfeld, J.L., Weissfeld, L., Thaete, F.L., Kuller, L.H., McAdams, M., Lanza, E. \& Schatzkin, A. (2004) Relationship of visceral adipose tissue to recurrence of adenomatous polyps. Am. J. Gastroenterol., 99, 687-693.

Stocks, T., Lukanova, A., Johansson, M., Rinaldi, S., Palmqvist, R., Hallmans, G., Kaaks, R. \& Stattin, P. (2008) Components of the metabolic syndrome and colorectal cancer risk; a prospective study. Int. J. Obesity, 32, 304-314.

The Japan Diabetes Society. (2010) Treatment Guide for Diabetes 2010. Bunkodo, Ltd., Tokyo, Japan. 11-12. (in Japanese)

Trevisan, M., Lui, J., Muti, P., Misciagna, G., Menotti, A. \& Fucci, F.; Risk Factors and Life Expectancy Research Group. (2001) Markers of insulin resistance and colorectal cancer mortality. Cancer Epidem. Biomar., 10, 937-941.

Tsilidis, K.K., Brancati, F.L., Pollak, M.N., Rifai, N., Clipp, S.L., Hoffman-Bolton, J., Helzlsouer, K.J. \& Platz, E.A. (2010) Metabolic syndrome components and colorectal adenoma in the CLUE II cohort. Cancer Cause Control, 21, 1-10.

Yamamoto, S., Nakagawa, T., Matsushita, Y., Kusano, S., Hayashi, T., Irokawa, M., Aoki, T., Korogi, Y. \& Mizoue, T. (2010) Visceral fat area and markers of insulin resistance in relation to colorectal neoplasia. Diabetes Care, 33, 184-189.

Yui, H.Y., Whittemore, A.S. \& Shibata, A. (2004) Increasing colorectal cancer incidence rates in Japan. Int. J. Cancer, 109, 777-781.

Wang, Y.Y., Lin, S.Y., Lai, W.A., Liu, P.H. \& Sheu, W.H. (2005) Association between adenomas of rectosigmoid colon and 
metabolic syndrome features in a Chinese population. $J$. Gastroenterol. Hepatol., 20, 1410-1415.

Winawer, S.J., Zauber, A.G., O'Brien, M.J., Ho, M.N., Gottlieb, L., Sternberg, S.S., Waye, J.D., Bond, J., Schapiro, M., Stewart, E.T., Panish, J., Ackroyd, F., Kurtz, R.C., Shike, M. \& the National Polyp Study Workgroup. (1993) Randomized comparison of surveillance intervals after colonoscopic removal of newly diagnosed adenomatous polyps. N. Engl. J. Med., 328, 901-906.

Zarchy, T.M. \& Ershoff, D. (1994) Do characterisctics of adenomas on flexible sigmoidoscopy predict advanced lesions on baseline colonoscopy? Gastroenterology, 106, 1501-1504. 